\title{
Terminologia tachigrafica in alcune similitudini del De virginitate di Basilio d'Ancira
}

\author{
Giovanna Menci
}

I papiri tachigrafici sono soltanto 76 su un totale di quasi 16.000 papiri letterari ${ }^{1}$; il loro numero è aumentato notevolmente negli ultimi 50 anni, perché questi testi, per lo più frammentari e di difficile interpretazione, hanno suscitato la curiosità e l'interesse degli studiosi. Purtroppo sia le edizioni dei papiri, sia gli studi sui vari aspetti della cosiddetta tachigrafia, scritti nelle varie lingue, mostrano una terminologia non sempre uniforme. La parola tachigrafia non è la sola ad essere impiegata per definire questo sistema rapido di scrittura tramite segni che ci è stato restituito dai papiri. Per esempio, in tedesco, oltre a Tachygraphie, si usano i termini Kurzschrift o Schnellschrift; in inglese, oltre a tachygraphy, si usano altri due termini, shorthand e stenography. Si fa dunque riferimento a una scrittura veloce, abbreviata o stretta.

Nessuna di queste parole, tuttavia, rende l'idea di quella che era la tecnica di base del sistema usato nell'Egitto romano e bizantino per prendere appunti da

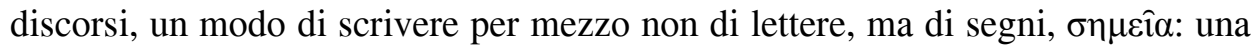
lettera si poteva rappresentare con uno o due tratti caratteristici della stessa, mentre una sillaba o una parola corrispondevano a una combinazione di tratti. I segni sillabici erano alla base dei segni più complessi del cosiddetto commentario tachigrafico (da commentarium o commentum, nel senso di testo

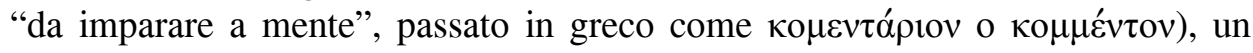
elenco di parole raggruppate per tetradi sulla base di associazione di idee.

Sui termini sillabario e commentario c'è concordanza fra gli studiosi; essi

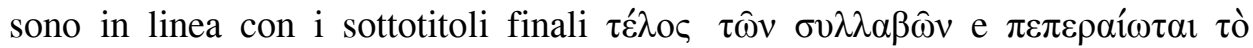

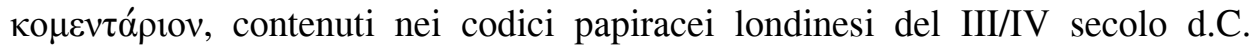
pubblicati da Milne nel $1934^{2}$. Per quanto riguarda invece il nome di quell'attività che i papirologi hanno chiamato tachigrafia, Kurzschrift, stenography, ecc., non si trovano suggerimenti nei papiri; tachigrafia è una

\footnotetext{
${ }^{1}$ Così risulta da una ricerca eseguita nel LDAB <http://www.trismegistos.org/ldab/search.php> tramite la chiave "tachygraphy" nel campo "Genre". Nel catalogo dei papiri letterari di Oldfather del 1923 (Oldfather, Texts) questi testi erano assenti. Nella prima edizione del catalogo del Pack, Texts, del 1952, erano soltanto 20 (p. 92), nella seconda, del 1965, 27 (p. 141).
}

${ }^{2}$ Milne, Shorthand. LDAB 5532 e 5533. 
parola d'invenzione più recente, che si basa sull'esistenza del termine

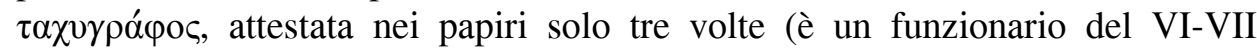
secolo) e poi presente in autori dall'VIII secolo in poi. A quest'epoca

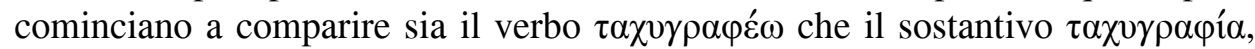
con riferimento all'attività dello scrivere velocemente. Velocemente, ma non per

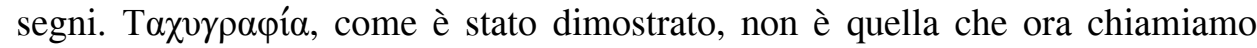
tachigrafia, tachygraphy, tachygraphie o Tachygraphie ${ }^{3}$; è in realtà la scrittura minuscola (contrapposta alla $\kappa \alpha \lambda \lambda \imath \rho \alpha \varphi$ í $\alpha$ ), nella quale l'uso di abbreviazioni convenzionali da parte di chi scrive, il $\tau \alpha \chi v \gamma \rho \alpha ́ \varphi \varsigma$, , è del tutto occasionale, in quanto investe solo la fine di qualche parola o intere parolette ricorrenti di

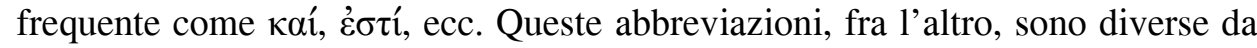
quelle della cosiddetta tachigrafia dei papiri. Se ne deduce che il termine 'tachigrafia' e l'aggettivo 'tachigrafici' riferito ai papiri non sono appropriati, benché essi siano in uso fin dalle prime edizioni e i primi studi sull'argomento.

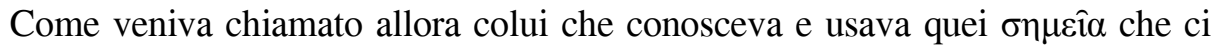
vengono restituiti dai papiri cosiddetti tachigrafici? È molto semplice:

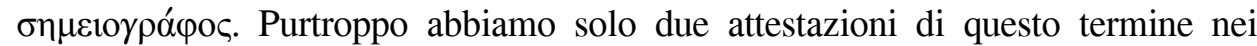
papiri; una di esse è chiarissima e indubitabile, perché è contenuta in un contratto di apprendistato, P.Oxy. IV 724, stipulato il $1^{\circ}$ marzo del 155 d.C. Uno schiavo

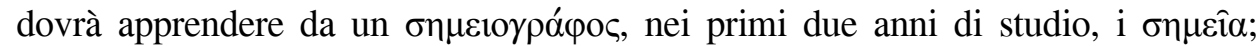

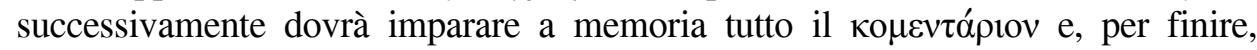
dovrà scrivere e interpretare con i segni ogni tipo di linguaggio quotidiano.

Quello che i papiri non ci dicono è sia il nome di questa attività, sia la denominazione dei vari elementi che compongono l'unità minima del commentario tachigrafico, la tetrade di parole. $\mathrm{Ci}$ viene però in aiuto un passo di un trattato sulla verginità, trasmesso sotto il nome di San Basilio, indirizzato a un certo Letoio, vescovo di Melitene, il cui titolo completo è Sull'autentica

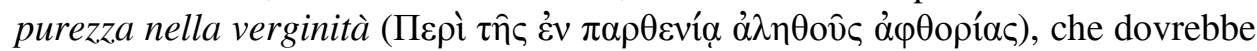
essere stato composto tra il 356 e il 358. Siamo dunque nel IV secolo, un'epoca in cui la cosiddetta tachigrafia in Egitto è già molto diffusa. Milne, nell'introduzione alla sua edizione del commentario, aveva riportato il brano solo parzialmente ${ }^{4}$ e non aveva considerato la possibilità di istituire un confronto tra la terminologia usata nel passo e la realtà 'editoriale', potremmo dire, che ci presentano i cosiddetti papiri tachigrafici. La mia analisi si propone di individuare o, meglio, istituire una corrispondenza fra la terminologia all'interno

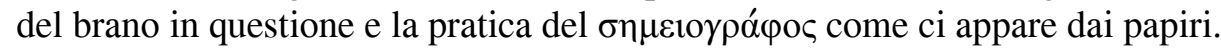

La paternità di Basilio di Cesarea, S. Basilio Magno, è stata messa in dubbio fin dal '700, ma fu soltanto all'inizio del secolo scorso che Ferdinand Cavallera propose di attribuire il trattato a Basilio d'Ancira, di cui si sapeva (da Girolamo,

\footnotetext{
${ }^{3}$ Cfr. Luzzatto, Grammata, 12-14 e passim.

${ }^{4}$ Milne, Shorthand, 4.
} 
Vir. Ill. 89) che era stato medico e vescovo omoiusiano e aveva composto un De virginitate $^{5}$. I motivi principali di questa attribuzione sono sia la dedica al vescovo Letoio, col quale il collega di Ancira condivideva le opinioni omoiusiane sulla natura di Cristo, sia la presenza di dettagli fisiologici abbondanti, precisi, alcuni dei quali ritenuti scabrosi, riguardo alla verginità e al pericolo rappresentato dalla frequentazione degli eunuchi, dettagli che, se scritti da un ex medico come Basilio d'Ancira, risulterebbero meno sconcertanti di quanto lo sarebbero se scritti da Basilio di Cesarea.

Il testo greco di riferimento è tuttora quello di Migne, affiancato dalla traduzione latina nel vol. XXX della Patrologia Graeca $(\text { coll. } 733-734)^{6}$, che è praticamente lo stesso dell'edizione parigina di Garnier del 1839; manca ancora purtroppo un'edizione critica del trattato. Ovviamente non è questa la sede per esaminarne l'intero contenuto ${ }^{7}$ e i motivi stilistici che si oppongono all'attribuzione a Basilio Magno. Accennerò soltanto al fatto che l'intento didattico dell'autore si riflette in uno stile denso di locuzioni esplicative, come risulta evidente anche solo da questo brano ridondante di similitudini, attraverso le quali l'autore vuole dimostrare che ogni pensiero, anche se non si realizza, rimane impresso nell'anima. Fra le similitudini, ben quattro, riguardando da vicino la cosiddetta tachigrafia, possono essere utili per individuare una terminologia moderna appropriata, visto che la tecnica a cui accenna l'autore trova riscontro nei manuali, negli esercizi, negli appunti che ci restituiscono i papiri.

${ }^{5}$ Cavallera, De Virginitate. Sulla tradizione manoscritta e le successive edizioni del testo, si veda Leroy, Tradition. Relativamente a questo passo, ho consultato la traduzione francese di Coudreau (Coudreau/Miquel, Intégrité, 58-60). Quella tedesca di Anne Burgsmüller (Burgsmüller, Askeseschrift) purtroppo non è stata molto utile, visto che non è stato compreso che nel brano si parla di tachigrafia. Quasi inutilizzabile il confronto con la traduzione francese da una versione paleoslava del $\mathrm{X}$ secolo, perché in questa versione si riscontrano lacune molto estese e fraintendimenti del greco (Vaillant, De Virginitate).

${ }^{6} \mathrm{~L}$ 'intero trattato è in Migne, $P G \mathrm{XXX}, 669-810$.

7 Tento soltanto una sintesi accennando a quelli che sono i punti principali. La verginità, sostiene Basilio, rende simili all'incorruttibilità di Dio; poiché procede dall'anima verso il corpo, la vergine deve custodire l'anima pura, vincendo l'attrazione verso l'altro sesso e il piacere dei sensi. Tuttavia ogni mortificazione fisica è controproducente perché, indebolendo il corpo, fa ammalare anche l'anima; la vera verginità si deve basare dunque su un rapporto armonioso fra anima e corpo. Perciò Basilio sostiene che il matrimonio è lecito, opponendosi così all'encratismo, cioè la continenza rigorosa e mortificante; riprendendo la teoria platonica dell'androginia originaria, considera l'istinto sessuale come il desiderio, insito nella natura umana, di ripristinare l'unione con l'altro sesso. La verginità è dunque da considerare un superamento delle tendenze naturali umane, frutto di una scelta, non un dovere prescritto da Dio. Condanna l'autocastrazione perché con l'impotenza fisica il desiderio non si annulla, ma si moltiplica; le vergini pertanto, secondo Basilio, dovranno fare attenzione agli eunuchi. Infine si giustifica per essere entrato in particolari scabrosi riguardo alla natura maschile, dicendo che le vergini non sono certo delle bambine; è vero che, secondo il precetto evangelico, devono essere semplici come colombe, ma devono anche essere prudenti come serpenti. 
Dopo aver espresso il concetto che un pensiero insito nell'anima è come una scrittura impressa "con i colori e le dimensioni dell'immaginazione e ancora con le figure che la mente ha tratteggiato in lei"", Basilio passa a una similitudine più complessa e articolata, che ha come primo termine di paragone l'aspetto dell'anima

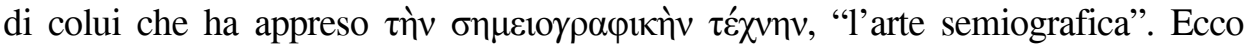
dunque come veniva chiamata quella che oggi si definisce tachigrafia; i papiri tachigrafici dovrebbero essere più propriamente definiti semiografici.

Questo è il testo della prima similitudine, in cui vengono nominati gli elementi strutturali dell'apprendimento della semiografia:

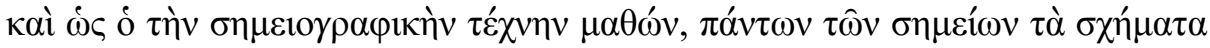

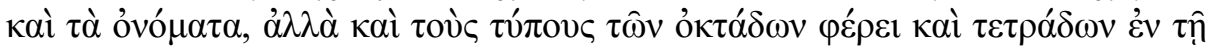

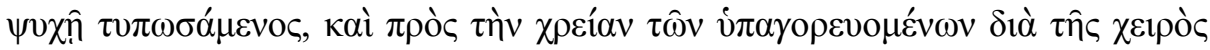

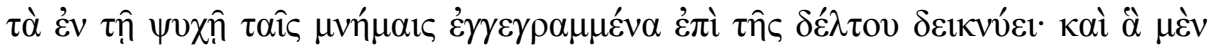

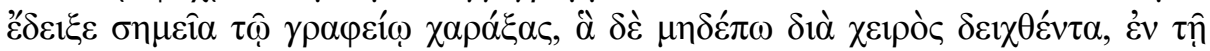

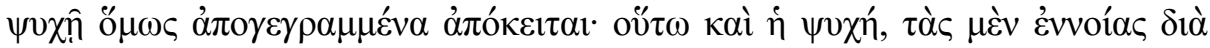

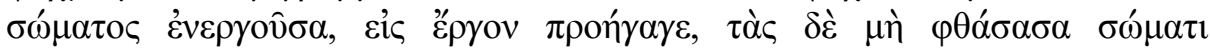

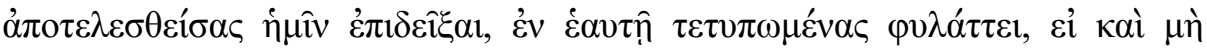

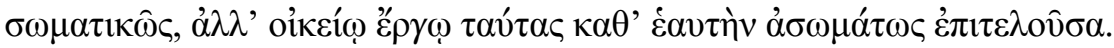

E come colui che ha appreso l'arte semiografica porta, avendole impresse nell'anima, le figure e i nomi di tutti i segni, nonché le forme delle ottadi e delle tetradi, e a seconda di ciò che viene dettato raffigura con la sua mano sulla tavoletta quanto sta scritto nell'anima con il ricordo - e sia quei segni che ha mostrato incisi con lo strumento scrittorio, sia quelli che non sono stati mostrati con la mano rimangono comunque trascritti nell'anima -; così anche l'anima, esprimendo pensieri tramite il corpo, li ha portati ad azione, e quelli che non è arrivata a mostrarci realizzati dal corpo, li custodisce in se stessa incisi, anche se non corporalmente in modo intimo, realizzandoli tra sé immaterialmente.

Si istituisce dunque un paragone fra i pensieri insiti nell'anima e le figure e $\mathrm{i}$ nomi di tutti i segni, nonché le forme delle ottadi e delle tetradi che risiedono

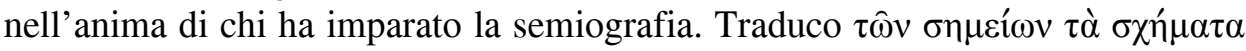
"le figure dei segni" sulla falsariga della traduzione latina notarum figuras in PG. Ma che cosa sono $\tau \grave{\alpha}$ òvó $\mu \alpha \tau \alpha$ ? In un sillabario di solito i segni si succedono incolonnati e sono affiancati dalla sillaba a cui corrispondono; inoltre un sillabario può contenere i segni per preposizioni, articoli, pronomi ${ }^{9}$. Credo che

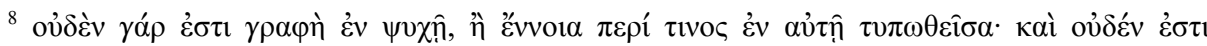

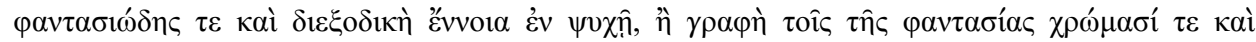

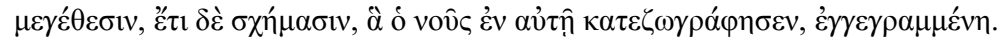

${ }^{9}$ Milne, Shorthand, 3 e 13-18.
} 
gli ỏvó $\mu \alpha \tau \alpha, i$ "nomi" dei segni, siano le lettere, le sillabe, le parole, equivalenti ai segni, tutto ciò a cui di solito si fa riferimento con 'spiegazioni' dei segni o 'scrittura per esteso' o con espressioni simili.

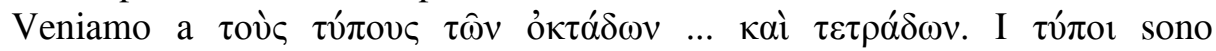
probabilmente le forme che restano incise nella memoria come delle impronte. Qualcosa di diverso rispetto alle figure dei segni del sillabario, cioè gli $\sigma \chi \eta ́ \eta \alpha \tau \alpha$ ? Non sappiamo quale tipo di sistema tachigrafico o, meglio, semiografico fosse in uso ad Ancira, in Galazia, perché non abbiamo, che io sappia, documentazione diretta di una scrittura del genere proveniente né da questa città né dal resto dell'Asia Minore, ma che vi fosse un'analogia con il sistema in uso in Egitto, questo testo di Basilio mi sembra dimostrarlo. È sorprendente trovare, a oltre $2000 \mathrm{~km}$ di distanza, riferimenti a un sistema che è sempre stato ritenuto

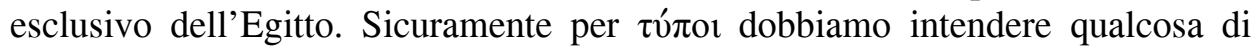
diverso dagli $\sigma \chi \eta ́ \eta \alpha \tau \alpha$, visto che più avanti entrambi i termini vengono citati in riferimento a una ottade, un gruppo di otto parole. Nell'ultima similitudine si

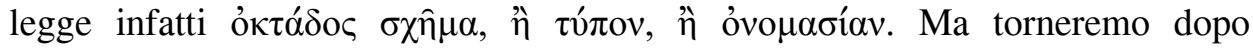
sull'individuazione dei $\tau v ́ \pi o l$, anche perché il termine ricorre altre due volte.

Procediamo con la lettura. Basilio pone sullo stesso piano di giudizio i pensieri peccaminosi insiti nell'anima e quelli portati a compimento dal corpo:

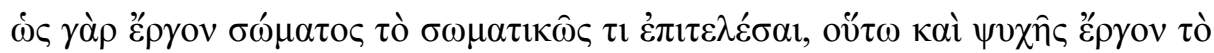

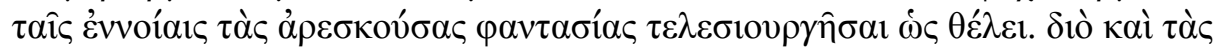

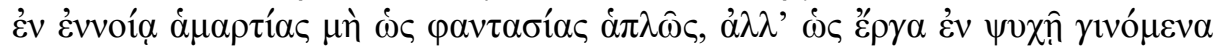

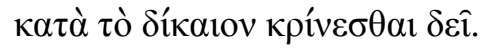

Perché, come è opera del corpo il realizzare qualcosa corporalmente, così è opera dell'anima il dar compimento con i pensieri, come vuole, alle fantasie che le piacciono. Pertanto anche i peccati che risiedono nel pensiero vanno giudicati non come semplici immaginazioni, ma, equamente, come opere attuantisi nell'anima.

A questo punto mette a nudo l'anima, con una curiosa immagine che ce la

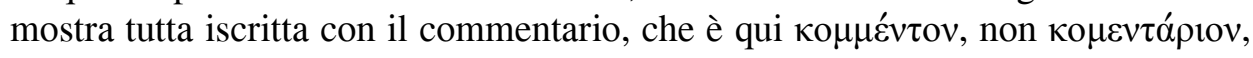
come nei papiri:

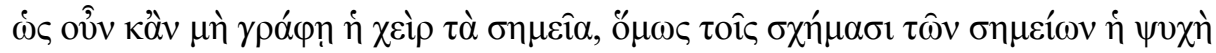

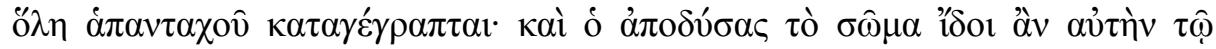

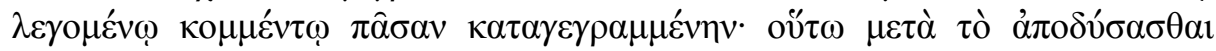

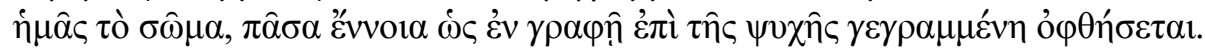

Come dunque, anche se la mano non scrive i segni, tuttavia l'anima nel suo complesso è riempita dovunque dalle figure dei segni, e chi si spogliasse del 
corpo la vedrebbe tutta iscritta con il cosiddetto commentario, così dopo che ci saremo spogliati del corpo, ogni pensiero si vedrà tracciato come per iscritto nell'anima.

Prosegue con altre due similitudini. Nella prima istituisce un confronto fra i dei segni e le $\varphi \alpha v \tau \alpha \sigma i ́ \alpha$ dei peccati e dei pensieri che risiedono nell'anima:

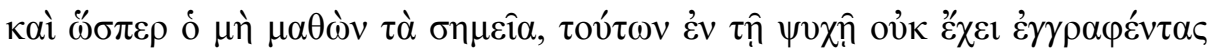

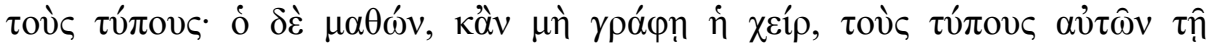

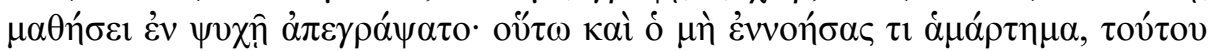

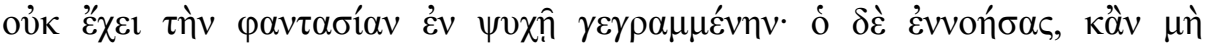

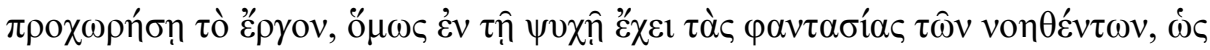

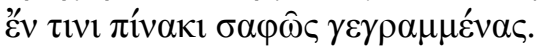

E come chi non ha imparato i segni, non possiede iscritti nell'anima le loro forme, e chi li ha imparati, anche se la mano non scrive, con l'apprendimento ha iscritto nell'anima le loro forme, così anche chi non ha pensato un peccato non ha di questo l'immagine iscritta nell'anima, e chi lo ha pensato, anche se non procede l'azione, tuttavia ha nell'anima le immagini di quello che ha pensato riprodotte chiaramente come in un quadro.

Infine la soluzione che porta alla purificazione dell'anima: dimenticare tutto, trascurando l'esercizio:

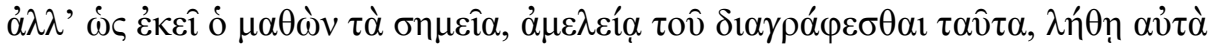

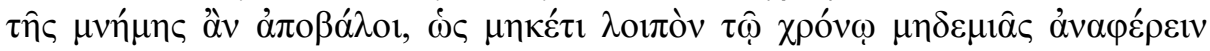

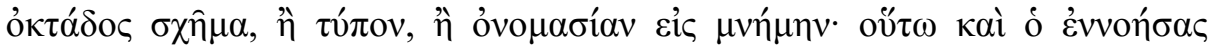

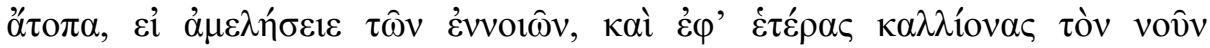

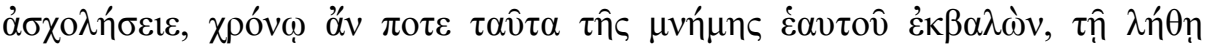

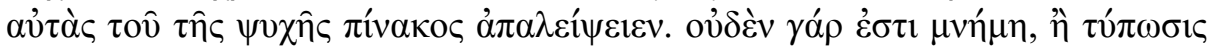
$\dot{\varepsilon} v v o t \omega \hat{\omega} v, \dot{\omega} \varsigma \dot{\varepsilon} v \psi v \chi \hat{n}, \dot{v} \pi \circ \zeta \hat{\omega} \gamma \rho \alpha \varphi \circ \varsigma$.

Ma, come nel primo caso, colui che ha appreso i segni, trascurando di scriverli, li perderebbe dalla memoria per dimenticanza, sicché col tempo non rimarrebbe nessuna possibilità di richiamare alla memoria né la figura, né la forma, né la denominazione di nessuna ottade, così anche chi ha pensato qualcosa fuori dalle regole, se trascurasse di pensarlo e dedicasse la mente ad altre cose più belle, col tempo, perdendo dalla propria memoria quelle cose, con la dimenticanza le cancellerebbe dal quadro dell'anima. Perché la memoria altro non è che, per così dire, l'impressione delineata nell'anima di pensieri.

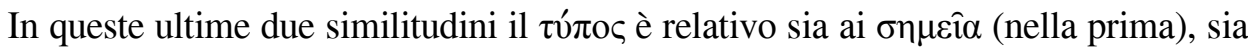


all'ottade (nella seconda). Non si nomina la tetrade, forse per distrazione. Riferiti

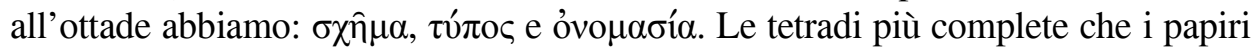
egizi ci hanno restitutito appaiono in effetti composte da vari elementi.

Secondo l'uso ormai divenuto comune, si distingue un 'segno principale', distintivo della tetrade, accompagnato da un 'elemento principale', scritto per esteso, a cui il segno corrisponde (una sillaba, una parola o un gruppo di parole); a fianco si colloca un elenco di quattro o più parole unite da associazione di idee o formanti una frase, e, infine, attorno al segno principale, sono tracciati dei 'segni secondari', semplici segni sillabici corrispondenti alla parte finale di ogni parola elencata nella tetrade ${ }^{10}$. Ogni volta che uno studioso moderno si è trovato a scrivere sulle tetradi del commentario, ha usato più o meno questa terminologia per indicare le varie parti della tetrade, seppure con lievi differenze nelle varie lingue.

Con questa accurata descrizione di Basilio, quasi anatomica direi, abbiamo la possibilità di sapere come gli antichi chiamavano le parti della tetrade o ottade: direi che la ỏvo $\mu \alpha \sigma i ́ \alpha$, cioè la denominazione, o anche nome, è quello che fin qui è stato chiamato l' 'elemento principale' in scrittura per esteso; lo $\sigma \chi \hat{\eta} \mu \alpha$ è il 'segno principale', la figura del segno che rappresenta la óvo $\mu \alpha \sigma i ́ \alpha$, mentre il

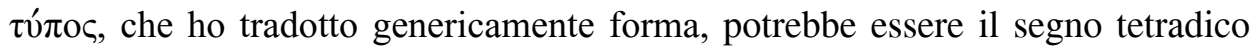
nella sua completezza, circondato cioè dai piccoli segni per le finali delle parole, oppure l'intera tetrade o ottade comprensiva di segni e parole. In entrambi i casi,

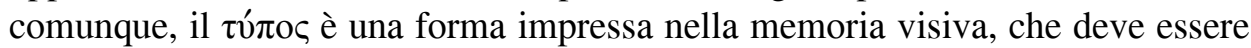
memorizzata perché le parole possano essere ricordate e scritte dal semiografo col minore dispendio possibile di tempo ${ }^{11}$.

Sorprende che Basilio dia importanza all'ottade. Fino a poco tempo fa, nei papiri, ne conoscevamo soltanto una (P.Ant. I 4), che era un ampliamento della pentade 200 dei commentari londinesi, con parole incolonnate ${ }^{12}$. Solo recentemente, nella revisione di PSI Congr.XXI 5, foglio di codice papiraceo del VI secolo proveniente da Antinoupolis ${ }^{13}$, contenente tetradi (o pentadi) nella prima colonna e tetradi nella seconda, mi sono accorta che le parole della seconda colonna sono sinonimi dei termini contenuti nella prima. Potrebbe essere questa l'ottade di cui parla Basilio, cioè una tetrade estesa con l'aggiunta di un'altra tetrade formata da sinonimi. Resta comunque il fatto che prendere l'ottade del commentario e tutti gli altri elementi correlati come termini di paragone per spiegare che i peccati contro la verginità restano impressi nell'anima significa che l'arte semiografica doveva essere non del tutto sconosciuta in ambito ecclesiastico,

${ }^{10} \mathrm{Si}$ vedano, per esempio, Pl. I e II in Milne, Shorthand, dove sono rappresentati due fogli dei codici londinesi contenenti tetradi del commentario tachigrafico, complete di segni.

${ }^{11}$ Non vedo la necessità di tradurre $\tau$ $v ́ \pi \circ \zeta$ in tre modi diversi nelle tre similitudini, come ha fatto Coudreau, "empreinte", "marque", "tracé" (Coudreau/Miquel, intégrité, 58-60).

${ }^{12}$ Così la descrive Milne, Shorthand, 30, in apparato.

${ }^{13}$ Menci, Revisione. 
anzi direi piuttosto diffusa, anche fuori dall'Egitto e con caratteristiche del tutto simili a quella testimoniata nei papiri ${ }^{14}$.

\section{Bibliografia}

Burgsmüller, Askeseschrift $=$ Anne Burgsmüller, Die Askeseschrift des Pseudo-Basilius: Untersuchungen zum "Über die wahre Reinheit in der Jungfräulichkeit”, Tübingen 2005.

Cavallera, De Virginitate = Ferdinand Cavallera, Le "De Virginitate" de Basile d'Ancyre, "Revue d'Histoire Ecclésiastique" 6 (1905), 5-14.

Coudreau/Miquel, Intégrité = Claude Coudreau / Pierre Miquel, De la véritable intégrité dans la virginité, Saint-Benoît 1981.

Leroy, Tradition = François Joseph Leroy, La tradition manuscrite du "de virginitate" de Basile d'Ancyre, "Orientalia Christiana Periodica" 38 (1972), 195-208.

Luzzatto, Grammata = Maria Jagoda Luzzatto, Grammata e syrmata. Scrittura greca e produzione libraria tra VII e IX secolo, AnPap 14-15 (2002-03) [2005], 1-89.

Menci, Revisione = Giovanna Menci, Revisione di PSI Congr.XXI 5: commentario tachigrafico con oktades?, in: A. Casanova / G. Messeri / R. Pintaudi (edd.), E sì d'amici pieno. Omaggio di studiosi italiani a Guido Bastianini per il suo settantesimo compleanno, (Pap.Flor. 45) Firenze 2016, I, 253-9.

Migne, $P G$ = Jacques-Paul Migne, Patrologiae Cursus Completus. Series Graeca, 1-161, Parisiis 1857-66.

Milne, Shorthand = Herbert J. M. Milne, Greek Shorthand Manuals, Syllabary and Commentary, London 1934.

Oldfather, Texts $=$ Charles H. Oldfather, The Greek Literary Texts from Greco-Roman Egypt: A Study in the History of Civilization, Madison 1923.

Pack, Texts = Roger A. Pack, The Greek and Latin Literary Texts from Greco-Roman Egypt, Ann Arbor 1952; Second revised and enlarged ed., Ann Arbor 1965.

Reynard, Médecine = Jean Reynard, Médecine et théologie selon Basile d'Ancyre, in: V. BoudonMillot / B. Pouderon (edd.), Les Pères de l'Église face à la science médicale de leur temps, Paris 2005, 105-20.

Vaillant, De Virginitate $=$ André Vaillant, De Virginitate de Saint Basile. Texte vieux-slave et traduction française, Paris 1943.

${ }^{14}$ Nella letteratura superstite questo passo potrebbe rappresentare soltanto la punta di un iceberg. I trattati fisiologici dei Padri della Chiesa sono stati spesso trascurati, perché giudicati o troppo teologici o troppo medici. Esistono studi recenti che li prendono in considerazione non soltanto come volgarizzazioni delle dottrine elaborate dalle differenti scuole di medicina, ma anche in relazione con la teologia, l'antropologia e l'etica. Su medicina e teologia in Basilio di Ancira, si veda Reynard, Médecine. 